\title{
La divulgación científica en ciencias sociales a través de las exposiciones: un estudio de caso.
}

\author{
Isabel SolANAS GARCÍA \\ Universidad Ramon Llull (España) \\ isabelsolanasgarcia@gmail.com \\ Cristina Martorell Castellano \\ Universidad Ramon Llull (España) \\ cristinamc4@blanquerna.url.edu \\ Carolina SERra FolCH \\ Universidad Ramon Llull (España) \\ carolinasf@blanquerna.url.edu
}

\section{Resumen}

El presente artículo aborda algunas de las vías que permiten divulgar los resultados de trabajos de investigación entre sectores de la población más amplios que los estrictamente académicos o profesionales. A través de un estudio de caso, la exposición "Publicidad en Cataluña 1857-1957. Roldós y los pioneros", se recorre el proceso de adaptación de un trabajo académico a un producto divulgativo.

Palabras clave: divulgación científica, publicidad, exposición.

\section{Scientific dissemination in social sciencies. A case study.}

\begin{abstract}
This article focuses on some of the ways that allow researchers to disseminate the results of their academic projects among society, that is to say, beyond professional or academic sectors. Through a case study, the exhibition "Advertising in Catalonia 1857-1957. Roldós and the pioneers", we will revise the adaptation process from an academic study to an informative content.
\end{abstract}

Key Words: scientific dissemination, advertising, exhibition.

\section{Referencia normalizada:}

Solanas García, I.; Martorell Castellano, C. y Serra Folch, C. (2013) La divulgación científica en ciencias sociales a través de las exposiciones: un estudio de caso. Historia y Comunicación Social. Vol. 18. $\mathrm{N}^{\mathrm{o}}$ Especial Noviembre. Págs. 815-826.

Sumario: 1. Introducción. 2. Marco teórico: la divulgación científica 3. El proceso de adaptación: de un trabajo académico a un proyecto divulgativo. 3.1 La adaptación del contenido. 3.2 La adaptación formal. 4. El proceso de difusión de la investigación. Campaña publicitaria y actividades complementarias. 5. Resultados del proyecto. 6. Conclusiones. 7. Referencias bibliográficas. 8. Notas 


\section{Introducción}

En el ámbito de las ciencias sociales, y hablando en términos generales, la cuestión de la difusión y la divulgación del conocimiento no parece plantear una complejidad excesiva. Su debate académico invita a un diálogo colaborativo que permita explorar y compartir posibles fórmulas para llevar a cabo esta tarea, tanto en sus aspectos de planteamiento y ejecución como en lo relativo a la obtención de los recursos que las hagan viables. Las ciencias sociales abarcan un abanico de temas tan variados que invitan a pensar en términos de amplitud y reciprocidad de intereses. La voluntad de saber más, en este caso, parece fácil de compartir. Si las ciencias sociales nacen y se nutren del estudio de múltiples cuestiones, coloquialmente hablando, vinculadas a la sociedad, es lógico pensar que esa misma sociedad se mostrará receptiva a sus logros, siempre que éstos sean presentados adecuadamente. El estudio de la comunicación periodística o de la comunicación comercial y publicitaria, a las cuales está mayoritariamente expuesta la sociedad en su conjunto, serían ejemplos de este universo de intereses compartidos. De ahí que, simplificando al máximo y partiendo de un contenido que a priori sea capaz de despertar interés en la sociedad, se trataría de acertar en la selección de los ingredientes y en el cálculo de sus proporciones, de manera que fuera bien recibido por el paladar y por los hábitos de ese potencial público consumidor de productos culturales. Una vez logrado esto, el acierto en un buen envasado y presentación junto con unas condiciones favorables de accesibilidad al público, apuntarían a su mantenimiento en el mercado y, en el mejor de los casos, a un notable éxito comercial. Aunque sabemos que las circunstancias reales superan esta simplicidad, el símil sigue siéndonos útil a efectos didácticos.

La exposición "Publicidad en Cataluña 1857-1957. Roldós y los pioneros", comisariada por las tres autoras de este artículo junto con el Dr. Joan Sabaté López, ejemplifica un caso de traslación de un trabajo de investigación doctoral a un proyecto con vocación divulgativa. A lo largo de este artículo, y tomando como ejemplo esta muestra, se pretende exponer el proceso de transformación de un trabajo académico en un producto de interés sociocultural -la exposición anteriormente mencionada-. Finalmente, y con el objeto de valorar el impacto social de la muestra, se presentarán los resultados y la repercusión social obtenida.

\section{Marco teórico: la divulgación científica}

Compartimos con el periodista y científico Manuel Calvo su consideración de la divulgación de la ciencia como el proceso mediante el cual se transmite "al gran público, en lenguaje accesible, decodificado, informaciones científicas y tecnológicas" (Calvo, 2006b). Se trata, por tanto, de establecer lazos o puentes que conecten dos ámbitos tradicionalmente alejados: la ciencia o la academia y la sociedad. Como argumenta Calvo, se trata de un proceso doble, ya que "por un lado, debe extraer su sustancia, sus materiales, del cerrado ámbito cientifico, y por otro, ha de llegar, 
interesar y, si es posible, entusiasmar al lector común con sus resultados" (Calvo, 1999:31). El significado del término divulgación pues, no sólo hace referencia a la acción de extender o difundir un conocimiento entre la población, sino también a la forma en la que se hace, puesto que estos conocimientos científicos deben presentarse de forma asequible e inteligible para esa población (Belenguer, 2003:45). Para llevar a cabo este proceso es necesario acercar el mensaje al receptor, por lo que se hace indispensable el uso de un lenguaje claro y conciso (Quiñónez, 2011:2; Pascuali, 1979). Adicionalmente, se añaden dos condicionantes esenciales para que la difusión de una idea pueda considerarse divulgación científica: a) que ésta no se realice en el marco de las enseñanzas regladas, y b) que su objetivo último no sea la formación de especialistas (Roqueplo, 1983:21, cfr. en Belenguer, 2006:45-46). Así, el divulgador científico asume una triple responsabilidad, que consiste en "informar, explicar y facilitar la comprensión" (Calvo, 1999:67). En el caso de la exposición "Publicidad en Cataluña 1857-1957. Roldós y los pioneros", esta triple responsabilidad se concreta en los siguientes objetivos: informar al público sobre la existencia de este producto cultural; explicar, tomando la figura de Rafael Roldós como hilo conductor, los principales acontecimientos que han ayudado a configurar la historia de la publicidad en nuestro país; y, finalmente, facilitar la comprensión del público mediante la estructuración de los contenidos de la muestra y la incorporación de objetos, material gráfico y multimedia.

\section{El proceso de adaptación: de un trabajo académico a un proyecto divulgativo.}

La Exposición "Publicidad en Cataluña 1857-1957. Roldós y los pioneros", celebrada en el Palau Robert de Barcelona entre el 7 de octubre del 2011 y el 1 de abril de 2012, constituye el eje central y vertebrador de un proyecto que perseguía acercar la actividad publicitaria a la ciudadanía. El origen de esta muestra se encuentra en la tesina de doctorado de la profesora Serra, titulada Rafael Roldós Viñolas, pionero de la publicidad en España (2009) y dirigida por el Dr. Joan Sabaté. La figura de Rafael Roldós Viñolas (1846-1918), hasta la fecha el primer agente publicitario documentado en España, constituyó el punto de partida tanto del trabajo académico de Serra como del posterior proyecto divulgativo.

La indagación bibliográfica realizada había puesto de manifiesto que las referencias a Rafael Roldós en el estudio de la historia de la publicidad en España eran escasas y que, en cualquier caso, sólo cubrían parcialmente su trayectoria profesional. El carácter pionero y emprendedor del personaje, así como el prestigio social que obtuvo en la Barcelona de la época, no quedaban reflejados y, sin embargo, su estudio invitaba a presentarlo como un ejemplo claro de un nuevo perfil profesional y empresarial que estaba emergiendo a finales del siglo XIX. Roldós ilustraba un cambio de paradigma y su figura era útil para representar la relevancia que estaba adquiriendo la publicidad en la comercialización de los productos y marcas. Rafael Roldós, como otros agentes coetáneos, se inició como corredor de anuncios y, del mismo modo que William 
Tayler o James Lawson en Gran Bretaña, Charles Louis Havas en Francia o Volney B. Palmer, John L. Hooper o Charles Duveyrier en Estados Unidos - entre muchos otros, y cada uno con sus particularidades- también él vio las posibilidades de negocio que se abrían en la convivencia entre las empresas publicitarias y periodísticas (Solanas, 2011:282-300). El campo de estudio en torno a la figura de Rafael Roldós se ampliaba y, desde la perspectiva académica, invitaba a ampliar la investigación y redimensionarla en el futuro proyecto de tesis doctoral de la profesora Serra. Por otra parte, desde la óptica divulgativa, Roldós $-\mathrm{y}$, tras su muerte, el testigo recogido por sus descendientes- merecía un reconocimiento también por parte de los barceloneses, puesto que se erigió como un empresario inquieto y un impulsor de los negocios que ayudaron en la construcción de una Barcelona moderna. Además de valorar la oportunidad de hacer un homenaje a este pionero, se contempló el atractivo de dos circunstancias adicionales. Por un lado, el hecho de que en el año 2011 -y hasta la fecha- la empresa publicitaria fundada por Roldós en 1872, el Centro de Anuncios Roldós y Compañía, siguiera operando en Barcelona de la mano de sus descendientes y bajo la denominación Roldós S.A. Por otro lado, podía fijarse una coincidencia temporal, ya que se cumplían 150 años de la profesionalización de la actividad publicitaria en Cataluña. Así, el proyecto también debía realzar el papel de Barcelona -y, por extensión, de Cataluña- en la construcción de la industria publicitaria moderna en nuestro país, utilizando como hilo conductor la figura de Rafael Roldós Viñolas.

Adicionalmente, el homenaje a este pionero también permitía realzar el papel de otros tantos publicitarios de la época que asentaron los cimientos de la teoría y la práctica profesional de la publicidad moderna en España. Algunos de ellos ya gozaban de renombre en los textos académicos, como era el caso de Pedro Prat Gaballí, pero otras figuras como Rafael Borí, José Gardó, Joan Aubeyzon o Malcolm Thomson -un tanto eclipsadas- podían, y debían, ser recuperadas. Estas circunstancias fueron nuevos incentivos para dar el impulso definitivo a una exposición con vocación abierta a diferentes sectores de la población.

Por el modo en el que fue concebida la exposición y la función general y sociocultural que ésta perseguía, debía tener un tratamiento de tipo documental, es decir, su estructuración debía estar "ligada al valor informativo o científico de los objetos" (Alonso y García, 2010:26), pero con el valor añadido de la novedad, ya que era la primera vez que en España se exponía este material, en su mayoría inédito, bien en calidad de original o de reproducción. Del mismo modo, el propósito de la exposición debía traducirse en un guión que sería fundamental para enmarcarla, como argumenta Hugues, "dentro de una narrativa más amplia" (Hugues, 2010:26). Una exploración previa de la información disponible sirvió de base para acotar cronológicamente el alcance de la exposición. La muestra debía ofrecer una visión panorámica -acorde a su propósito divulgativo- que reflejase el nacimiento y desarrollo de la profesión publicitaria en Cataluña, desde 1857 -año en el que Rafael Roldós inicia su actividad como corredor de anuncios, con tan sólo once años- hasta 1957, cuando la reciente llegada de la televisión marcaba un importante punto de inflexión en el negocio publi- 
citario. La cifra de cien años de recorrido podía actuar como un nuevo reclamo para su visita.

La viabilidad de la exposición y del proyecto divulgativo en su conjunto hacía imprescindible el apoyo de organizaciones públicas y privadas. Para ello se realizó un dossier de presentación que permitió describir el proyecto formalmente y mostrar diferentes oportunidades de desarrollo y vías de participación institucional. El documento elaborado, Rafael Roldós Viñolas: pioner de la publicitat a Barcelona i a Catalunya. 150 anys de publicitat, recogía los objetivos y características principales del proyecto, un detallado inventario de los materiales que se habían localizado y que podían ser exhibidos - una vez hechas las consultas oportunas a sus propietarios o depositarios-, y una propuesta de actividades complementarias, cuya viabilidad también dependía del apoyo económico e institucional que se alcanzase. El dossier incluía la propuesta de organización del trabajo a partir de los conocimientos del equipo de comisarios y argumentaba su capacidad para gestionar el proyecto. Asimismo, se incluía un listado de asesores y especialistas externos y de las instituciones públicas y privadas cuyo apoyo se pretendía obtener. Este último aspecto -la obtención de apoyo institucional- resultaba fundamental para poder materializar la exposición. Por último, se proponían acuerdos de patrocinio con algunas empresas y organismos del sector privado atendiendo a su dilatada trayectoria publicitaria. Tras diversas reuniones con las personalidades convenidas, se formalizó la colaboración de la Generalitat de Catalunya, a través de la Direcció d'Atenció Ciutadana i Difusió, que asumió la organización junto con la Facultad de Comunicación Blanquerna y el Ayuntamiento de Barcelona. Posteriormente, desde la esfera empresarial, se sumó Hewlett Packard, que vio en la exposición una forma de mostrar su innovadora tecnología de impresión. Anunciantes como Codorniu, Nestlé, Seat o Damm fueron contactados y aplaudieron la iniciativa, algunos de ellos prestándose a colaborar con la cesión de materiales de sus archivos. Había una razón para el proyecto, una oportunidad coyuntural, una estrategia para ejecutarlo y unos recursos para poder materializarlo. El proyecto de la exposición era, por tanto, un proyecto de trabajo real que ya avanzaba.

\subsection{La adaptación del contenido}

La divulgación científica requiere un doble proceso de adaptación. Por un lado, es necesario traducir el lenguaje científico - de carácter especializado o técnico- al registro estándar, con el objeto de facilitar su comprensión al público en general. Por otro lado, el divulgador científico debe realizar una labor de simplificación. Este proceso, como argumenta Calvo, siempre conlleva un cierto "factor de riesgo" (Calvo, 1999:15), en tanto que el divulgador opta deliberadamente por omitir cierta información en aras de lograr una mayor comprensión por parte de la audiencia. En el caso que nos ocupa, la exposición "Publicidad en Cataluña 1857-1957. Roldós y los pioneros", a las dificultades de plasmar los principales acontecimientos publicitarios a lo largo de un período de cien años había que añadir el desconocimiento del ciudadano medio de la temática de la exposición. Así, el discurso se enfocó a partir de una 
óptica general que fuera la antesala de una óptica más concreta y especializada, es decir, se optó por un tratamiento secuencial del discurso. De esta manera, por ejemplo, se consideró oportuno plasmar el contexto social de la Barcelona de finales del siglo XIX, para después mostrar la importancia que estaba adquiriendo la actividad anunciadora, a continuación dar paso a la figura del agente publicitario y, de la mano de éste, presentar la figura de Rafael Roldós y la fundación de su centro de anuncios.

Sin embargo, ese doble tratamiento -desde la generalidad hasta la especificidad y siguiendo una dinámica secuencial prefijada - no era suficiente para garantizar su comprensión, dada la amplitud del período que abarcaba la exposición -cien años, de 1857 hasta 1957- y el volumen de información que ello suponía. De este modo, se optó por dividir estructuralmente la muestra en tres períodos históricos ordenados cronológicamente: de 1857 a 1900, de 1901 a 1929 , y de 1930 a 1957. La secuencia temporal era útil para organizar la información pero también era una buena guía para orientar al visitante en su recorrido. En cada uno de esos tres bloques se desarrollaba una narración en tres niveles. El primer nivel situaba al espectador en el contexto histórico, político y social de cada uno de estos tres períodos; el segundo nivel se adentraba en el contexto publicitario catalán del momento -es decir, se hacía referencia a anunciantes, agencias, pioneros publicitarios o se mostraban anuncios destacados-, y el tercer nivel se reservaba al personaje de Rafael Roldós y a su actividad profesional.-De acuerdo con esta estructura, y en línea con las recomendaciones de Alonso y García, los textos de la exposición no eran "un listado de hechos o acontecimientos" sino que buscaban "explicar, guiar, motivar, informar y provocar la participación del visitante" (Alonso, García, 2010:146). Con este objetivo, se optó por incluir diversos plafones de texto en cada uno de los tres bloques cronológicos, haciendo uso de un código cromático y una tipografía que los diferenciaba con la finalidad, una vez más, de facilitar la comprensión del visitante. Lógicamente, esta estructuración del discurso multiplicó la necesidad de obtener material visual de diversa índole, para lo cual fue necesario establecer vínculos con diferentes organizaciones públicas y privadas (1). La búsqueda de nuevas fuentes de documentación permitió ejemplificar visualmente los tres niveles informativos anteriormente mencionados. Como criterio general, se recurrió al uso de abundante material visual de diferente naturaleza: piezas que habían formado parte del mobiliario y la decoración de la agencia, fotografías recuperadas que mostraban a algunos empleados en su lugar de trabajo, otras que mostraban las distintas ubicaciones de la agencia a lo largo del tiempo, además de numerosos bocetos de anuncios, documentos de carácter administrativo y de gestión y diversas actas notariales.

\subsection{La adaptación formal}

Las formas que puede adoptar el proceso de divulgación científica son múltiples: un museo, una exposición, un artículo en la prensa, un curso formativo, una demostración, un coloquio o un ciclo de conferencias son algunos ejemplos de esta variedad de alternativas formales. La exposición "Publicidad en Cataluña 1857-1957. Roldós y los pioneros", si atendemos al modelo propuesto por Raichvarg y Jacques (1991, 
cfr. en Belenguer, 2003:46), formaría parte de la llamada "divulgación en tres dimensiones", junto con los museos, los laboratorios populares, los viajes de divulgación o los gabinetes de historia natural, entre otros ejemplos. Estos autores distinguen cuatro formas de divulgación científica en función del lenguaje dominante de cada una de ellas. Así, además de la tipología anteriormente mencionada, la escritura es el código utilizado si la actividad divulgativa se realiza a partir de publicaciones en revistas, diarios o libros; la palabra es el lenguaje propio en el caso de elegir las conferencias, los cursos o los programas radiofónicos, mientras que la imagen es protagonista en la divulgación a través de dibujos, documentales y proyecciones audiovisuales.

En el caso que nos ocupa, la combinación de esos lenguajes facilitaba la modelación de la información y ampliaba las posibilidades de contemplar un diseño formal abierto pensando en términos creativos o de originalidad. La idea clave era que, desde la perspectiva formal, una exposición de carácter histórico no tenía por qué adoptar, necesariamente, un formato clásico sino que debía considerar elementos de diseño y presentación que le dieran un aspecto moderno, actual y atemporal. De ahí que, por ejemplo, se incluyeran pantallas de LEDS para la proyección continua de citas célebres (2) pronunciadas o escritas por algunos pioneros publicitarios. Del mismo modo, con el objetivo de mostrar la evolución de la publicidad durante el período que abarcaba la muestra (1857-1957), se decidió proyectar un vídeo que mostrase un anuncio publicado en cada uno de esos cien años. Así, en aproximadamente cuarenta minutos, los visitantes apreciaban los cambios formales y de contenido de estas gráficas publicitarias, a la vez que recordaban productos y marcas de antaño.

También se estudió el espacio asignado -la Sala 1 del Palau Robert-, compuesto por tres subsalas intercomunicadas. Cada uno de estos espacios fue ocupado por uno de los tres bloques estructurales anteriormente descritos y, de este modo, la secuencia cronológica también quedaba delimitada espacialmente, lo cual facilitaba su correcta comprensión por parte del público.

En esta fase de adaptación formal fue fundamental el esfuerzo de coordinación, control de calidad y vigilancia de timings entre los diferentes equipos de trabajo involucrados en el proyecto. El trabajo del equipo de comisarios debía armonizarse con las indicaciones y sugerencias del equipo del Palau Robert, el cual delegó en el estudio de diseño Porcuatro la propuesta de diseño y distribución de la exposición. Una vez aprobada esa propuesta general, Porcuatro confió a AloyMas su concreción y, posteriormente, a Grop Exposicions i Museografia S.L. la producción final y el montaje. La participación de Hewlett Packard se produjo en esta fase de reproducción de materiales. Haciendo uso de su avanzada tecnología, los materiales presentaban diferentes texturas y características. 


\section{EI proceso de difusión de la investigación. Campaña publicitaria y actividades complementarias.}

El carácter divulgativo de la exposición "Publicidad en Cataluña 1857-1957. Roldós y los pioneros" aconsejaba contemplar los principios básicos del marketing y la comunicación de eventos y de productos culturales. En este sentido, Siskind recuerda la conveniencia de considerar el hardware, o el evento en sí mismo, y el software o la estrategia para que el evento funcione (Siskind, 2007:s.p.). Una vez definido el hardware, se debía trabajar en el software -aunque, en realidad, en gran parte se hizo paralelamente dada la obligada interrelación entre ambos. La exposición requería contemplar una estrategia de lanzamiento pero también una estrategia de mantenimiento, pensando en su duración. Además, se partió de su consideración como producto noticioso - dándole un valor añadido de noticia- que podía nutrirse, periódicamente, de nuevos elementos que actuasen como recordatorio y mantuvieran su actualidad. A tal efecto, se diseñaron unas actividades adicionales que podían aumentar la dimensión de la exposición y, además, responder a intereses específicos de distintos públicos, ampliando así las posibilidades de ser visitada. Se trató, por tanto, de "cuidar informativamente y publicitariamente" (Alonso y García, 2010:212) la imagen de la exposición.

Con estas premisas, en relación al lanzamiento de la exposición, se procedió del siguiente modo. En primer lugar, se acordó la necesidad de realizar una campaña publicitaria que presentase a la ciudadanía la exposición. El medio elegido fue el exterior, a través del empleo de dos circuitos de opis y 2.200 banderolas en las principales calles barcelonesas. Para ello fue decisiva la ayuda del Ayuntamiento de Barcelona, a través del Departamento de Comunicación y Atención Ciudadana. En segundo lugar, se valoró la oportunidad de disponer de un escaparate de la exposición a pie de calle que pudiese despertar la curiosidad del paseante. De este modo se tuvo acceso a un espacio adicional en la entrada del Palau Robert que, a modo de reclamo, mostraba un despacho de agencia de publicidad antigua, utilizándose para ello parte del mobiliario de la época que conservaba la familia Roldós. Tanto en la recepción como en la entrada de la exposición, los visitantes encontraban un opúsculo de dieciséis páginas que explicaba la exposición, junto con un tarjetón que resumía la información básica de la muestra. En tercer lugar, se decidió aprovechar el inicio de la exposición para organizar un evento social: el acto inaugural, que contó con la presencia de autoridades políticas, medios de comunicación y líderes de opinión. Por último, en relación a la estrategia de mantenimiento, se acordó la realización de otras actividades de carácter complementario: la propuesta de hacer visitas guiadas a grupos organizados y la celebración de dos mesas redondas de carácter participativo y dirigidas preferentemente, en esta ocasión, a públicos especializados.

La campaña de lanzamiento se inició días antes de la inauguración con el envío de notas de prensa, tanto a los principales medios de comunicación generalistas como a aquéllos especializados en comunicación y publicidad. Estos medios fueron atendidos por los comisarios a lo largo de la mañana del día de la inauguración de la 
exposición y tuvieron oportunidad de visitarla pausadamente y hacer las preguntas oportunas.

Sin duda, el elemento principal de esta primera fase de lanzamiento fue el acto de inauguración, que tuvo lugar el 6 de octubre de 2011 y que contó con la presencia de figuras de alto reconocimiento en los ámbitos político, profesional y académico. El acto fue inaugurado por el señor Francesc Homs, secretario general de la presidencia y portavoz del gobierno de la Generalitat de Catalunya; el primer teniente de alcalde del Ayuntamiento de Barcelona, Joaquim Forn; el director general de atención ciudadana y difusión de la Generalitat de Catalunya, Ignasi Genovés; y el secretario de comunicación de la Generalitat de Catalunya, Josep Martí. Después de las intervenciones de estas personalidades, así como del Dr. Sabaté como representante del comisariado, se ofreció una copa de cava a los asistentes, por gentileza de Codorniu. Para comunicar el acto inaugural, se enviaron invitaciones a un amplio número de contactos seleccionados a partir de diferentes bases de datos. La apertura de la exposición también figuraba en la agenda cultural de algunos periódicos.

Por otro lado, en relación a la estrategia de mantenimiento, se realizó una nueva oleada de publicidad a principios del año 2012, una vez pasado el ecuador del periodo de exhibición: 800 banderolas fueron instaladas de nuevo como recordatorio de la exposición. A lo largo del período de exhibición de la muestra, los comisarios condujeron diversas visitas guiadas bajo petición de numerosos centros docentes de Cataluña. Finalmente, se organizó un ciclo de mesas redondas que, por un lado, pretendía tener un efecto recordatorio de la exposición y, por otro, apelaba al interés específico de diferentes públicos. La primera mesa estaba dirigida, principalmente, a profesionales del sector publicitario mientras que la segunda, primordialmente, se dirigía al entorno académico publicitario -estudiantes de publicidad, profesores e investigadores-. Ambas mesas redondas se ampararon bajo el título genérico "Publicidad en Cataluña. La herencia de los pioneros", y no sólo pretendían radiografiar el pasado y el presente de la publicidad en nuestro país, sino también debatir su futuro, a través del testimonio de algunos de sus protagonistas.

\section{Resultados del proyecto}

La exposición "Publicidad en Cataluña 1857-1957. Roldós y los pioneros" superó todas las expectativas, tanto en lo que respecta al número de visitas a la muestra como a la repercusión mediática que generó. Según los datos facilitados por el Palau Robert de Barcelona, un total de 67.461 personas visitaron la exposición a lo largo de los 201 días que se exhibió al público -esto es, desde el 6 de Octubre de 2011 hasta el 1 de Abril de 2012-. Ello implica un promedio de 10.069 visitantes mensuales o 336 diarios.

El balance de la repercusión mediática de esta muestra es igualmente positivo: más de catorce medios impresos -especialmente prensa generalista, revistas de ocio y 
revistas profesionales- $\mathrm{y}$ una veintena de medios digitales se hicieron eco del evento. A estas cifras se debe añadir el centenar de páginas web -institucionales, corporativas, de colegios profesionales, agendas culturales y blogs- que recomendaban o publicitaban la muestra, así como los comentarios que visitantes y entidades realizaban en las redes sociales, especialmente en Facebook y Twitter.

Un total de ocho programas televisivos y una emisora de radio -Ràdio 4- se interesaron por la exposición y solicitaron realizar un pequeño reportaje o entrevista a los comisarios de la muestra. Éste fue el caso de cadenas como Televisión Española (TVE), Canal 33, Barcelona Televisió o Badalona TV.

El impulso que otorgó la exposición a la figura de Rafael Roldós Viñolas contribuyó a que, unos meses después de que finalizara la exposición, fuera reconocido como "Miembro de Honor" de la Academia de la Publicidad, en un acto anual que reconoce la labor de los publicitarios más destacados de nuestro país.

\section{Conclusiones}

En este artículo se han revisado las principales etapas del proceso de divulgación científica en el marco de las ciencias sociales. A nuestro parecer, estas actividades divulgativas requieren dos esfuerzos fundamentales por parte de sus impulsores: por un lado, transformar estos hallazgos académicos en productos de interés para la sociedad en general -para lo cual resulta fundamental adaptarlos en forma y fondo a las inquietudes y conocimientos del público- $\mathrm{y}$, por otro lado, conseguir que estas actividades alcancen una cierta repercusión social, es decir, centrar también parte de los esfuerzos en la difusión de estas actividades.

La exposición "Publicidad en Cataluña 1857-1957. Roldós y los pioneros" ejemplifica cada una de las etapas y procesos de la divulgación científica en el ámbito de las ciencias sociales y, en base a los resultados obtenidos, puede considerarse un ejemplo de éxito de esta traslación de lo académico a lo divulgativo.

\section{Referencias bibliográficas}

ALONSO, Luis.; GARCÍA, Isabel (2010): Diseño de exposiciones. Concepto, instalación y montaje. Alianza Editorial. Madrid.

BELENGUER, Mariano (2003): "Información y divulgación científica: dos conceptos paralelos y complementarios en el periodismo científico", en Estudios sobre el Mensaje Periodístico, núm. 9, pp. 43-53.

CALVO, Manuel (1999): El nuevo periodismo de la ciencia. Quito: Ciespal. 
(2006a): "Objetivos de la divulgación científica". Artículo publicado en la web del periodista científico Manuel Calvo Hernando. Disponible en: http://www.manuelcalvohernando.es/articulo.php?id=66 [Consulta: 15/09/2013].

(2006b): "Conceptos sobre difusión, divulgación, periodismo y comunicación". Artículo publicado en la web del periodista científico Manuel Calvo Hernando. Disponible en: http://www.manuelcalvohernando.es/articulo.php?id=8 [Consulta: $15 / 09 / 2013]$.

HUGUES, Philip (2010): Diseño de exposiciones. Promopress. Barcelona.

QUIÑÓNEZ, Herly A. (2011). "Divulgación científica y tecnología: teoría y práctica periodística para la producción del documental", en Razón y Palabra, núm. 77 (agosto-octubre). Disponible en: http://www.razonypalabra.org.mx/varia/77\%20 5a\%20parte/70_Quinonez_V77.pdf [Consulta: 13/07/2013].

RAICHVARG, Daniel; JACQUES, Jean (1992). Savants et ignorants. Une histoire de la vulgarization des sciences. París: Seuil.

ROQUEPLO, Philippe (1983): El reparto del saber. Ciencia, cultura, divulgación. Barcelona: Gedisa.

SERRA, Carolina (2009). Rafael Roldós Viñolas, pionero de la publicidad en España. Tesina de doctorado presentada en la Universidad Ramon Llull.

SERRA, Carolina; MARTORELL, Cristina, SOLANAS, Isabel; SABATÉ, Joan (2012): Publicitat a Catalunya 1857-1957. Roldós i els pioners. Dossier de prensa. Universidad Ramon Llull. Barcelona.

SERRA, Carolina; SABATÉ, Joan; SOLANAS, Isabel; MARTORELL, Cristina (2011): Publicitat a Catalunya 1857-1957. Roldós i els pioners. Opúsculo de la exposición. Generalitat de Catalunya. Barcelona.

SERRA, Carolina; SABATÉ, Joan (2010): Rafael Roldós Viñolas: pioner de la publicitat a Barcelona i a Catalunya. 150 anys de publicitat. Dossier de presentación del proyecto. Grupo de Investigación en Estrategia y Creatividad Publicitarias, Universidad Ramon Llull. Barcelona.

SISKIND, Barry (2007): Marketing de eventos. Deusto. Barcelona.

SOLANAS, Isabel (2011): Orígenes de la Publicidad moderna (1800-1925). La aparición de la dirección y la gestión de cuentas como función profesional en las agencias de publicidad modernas. Tesis doctoral (Director: Dr. Joan Sabaté López). Universidad Ramon Llull. Barcelona.

\section{Notas}

1. Fondo de la familia Roldós, Archivo Fotográfico de Barcelona, Archivo Municipal Contemporáneo de Barcelona, Archivo General de la Universidad de Navarra, Biblioteca de Catalunya, Biblioteca del Pabellón de la República-CRAI (Universidad de Barcelona), Lahistoriadelapublicidad.com.

2. Algunas de las citas escogidas fueron: "Si el anuncio no contiene nada interesante, es tiempo perdido" (Rafael Borí y Josep Gardó, 1928); "No se anuncia sólo para vender, sino para vender con beneficios (Pedro Prat Gaballí, 1915); o "Hay una consideración de importancia primordial que es la siguiente: que los mercados no son lugares, sino que son personas (Malcolm Thomson, 1934). 


\section{Las autoras}

Isabel Solanas García es doctora en Publicidad por la Universidad Ramon Llull (2011) y licenciada en Publicidad y Relaciones Publicas por la Universidad Autónoma de Barcelona (1985). Es co-autora del libro, Dirección de cuentas. Gestión y planificación de cuentas en publicidad (Barcelona: UOC, 2008). Ha escrito diversos artículos académicos y también ha publicado en la revista sectorial Anuncios. Desde 1998 se dedica plenamente a la docencia universitaria. Con anterioridad desarrolló su trayectoria profesional en el área de cuentas. Trabajó en el instituto de investigación CIAC, y después lo hizo en las agencias J. Walter Thompson, como ejecutiva de cuentas y en Darcy Masius, Benton \& Bowles (DMB\&B), como supervisora y directora de cuentas.

Cristina Martorell Castellano es doctora en Comunicación (2013), Máster en Estrategia y Creatividad Publicitarias (2009) y Licenciada en Publicidad y Relaciones Públicas (2007) por la Universidad Ramon Llull. Desde 2011 es profesora de Publicidad en la Facultad de Comunicación Blanquerna (Universidad Ramon Llull). También es miembro del Grupo de Investigación en Estrategia y Creatividad Publicitarias. Anteriormente desarrolló su trayectoria profesional en el área creativa, trabajando de redactora publicitaria en la agencia BUM.

Carolina Serra Folch es doctoranda en Comunicación y Humanidades (DEA 2010) y licenciada en Publicidad y Relaciones Públicas (2002) por la Facultad de Comunicación Blanquerna de la Universidad Ramon Llull. Actualmente es investigadora del Grupo de Investigación en Estrategia y Creatividad Publicitarias en la Universidad Ramon Llull (2010) y profesora de la asignatura Sistemas y procesos de la publicidad en la UOC (2008), donde anteriormente ha trabajado como tutora de seguimiento (2008) y consultora de la asignatura Pràcticum (2008). También ha sido co-comisaria de la exposición "Publicidad en Cataluña (1857-1957). Roldós y los pioneros", y ha trabajado como ejecutiva de cuentas en las agencias de publicidad Concept/Advertising Soul, del grupo de comunicación BPMO (2010), y Vinizius Young \& Rubicam (2006). 